\title{
Índices de qualidade do solo sob diferentes sistemas de uso e manejo florestal e cerrado nativo adjacente ${ }^{1}$
}

\author{
Soil quality indexes under different forestry and adjacent native scrubland use and \\ management systems
}

\author{
Diego Antonio França de Freitas ${ }^{2 *}$, Marx Leandro Naves Silva ${ }^{3}$, Evaldo Luis $\operatorname{Cardoso}^{4}$ e Nilton Curi $^{3}$
}

\begin{abstract}
RESUMO - Existe uma dificuldade em quantificar diferentes atributos do solo que estão relacionados com a sustentabilidade e traduzir estes indicadores na forma de índices de qualidade do solo. Assim, objetivou-se neste trabalho estabelecer tais índices sob diferentes sistemas de uso e manejo florestal e cerrado nativo adjacente através da avaliação integrada de atributos físicos e químicos. Para isto, foram desenvolvidos dois índices de qualidade ( $\mathrm{IQS}_{1}$ e $\mathrm{IQS}_{2}$ ) utilizando um banco de dados sobre sistemas de manejo do eucalipto e cerrado nativo em Latossolos de Minas Gerais. No IQS ${ }_{1}$ os ecossistemas naturais, caracterizados pela mínima intervenção antrópica e de esperado equilíbrio, foram considerados como referência. $\mathrm{O} \mathrm{IQS}_{2}$ foi gerado a partir de um modelo aditivo que considera as funções principais do solo e os indicadores de qualidade a elas associados, sendo atribuídos pesos para as funções e indicadores. O IQS e $_{1}$ $\mathrm{IQS}_{2}$ apresentaram diferentes valores para os solos e sistemas de manejo analisados. Os sistemas sofreram reduções do IQS quando $_{1}$ comparados aos sistemas nativos, sendo que apenas os sistemas agrossilvopastoris apresentaram valores superiores de qualidade do solo quando comparados aos sistemas de referência. Pelo IQS ${ }_{2}$, os Latossolos Vermelho-Amarelos (LVA e LVA 2 ) apresentaram qualidade do solo superior aos Latossolos Vermelhos $\left(\mathrm{LV}_{1}\right.$ e $\left.\mathrm{LV}_{2}\right)$, sendo este resultado devido às menores limitações dos atributos químicos e físicos nas funções principais dos solos, influenciadas pelos sistemas de manejo daqueles solos. Apenas os sistemas agrossilvopastoris que utilizam eucalipto com arroz e eucalipto com soja proporcionaram melhoria da qualidade do solo pelos dois sistemas analisados.
\end{abstract}

Palavras-chave: Eucalipto. Solo-qualidade. Sustentabilidade.

\begin{abstract}
It is difficult to quantify the different soil characteristics that are related to sustainability, and to translate these indicators into soil-quality indexes. Therefore, this study aimed to establish such indexes for different forestry and adjacent native scrubland use and management systems by the integrated evaluation of physical and chemical properties. To this end, two quality indexes were developed (IQS and IQS $_{2}$ ), using a database of eucalyptus and native scrubland management systems for latosols in the state of Minas Gerais. In IQS ${ }_{1}$, natural ecosystems, characterized by minimal anthropic intervention and having the expected equilibrium, were used as reference. $\mathrm{IQS}_{2}$ was generated from an additive model that considers the main soil functions and the quality indicators associated with them by assigning weights to those functions and indicators. IQS ${ }_{1}$ and $\mathrm{IQS}_{2}$ presented different values for the soils and management systems analyzed. The systems suffered reductions in $\mathrm{SQI}_{1}$ when compared to the native systems, with only the agro-forestry systems having higher soil-quality values, when compared to the reference systems. Using IQS $\mathrm{I}_{2}$ the red-yellow latosols $\left(\mathrm{LVA}_{1}\right.$ and $\left.\mathrm{LVA}_{2}\right)$ showed higher soil quality than the red latosols $\left(\mathrm{LV}_{1}\right.$ and $\left.\mathrm{LV}_{2}\right)$, this result being due to the lower limitations of the chemical and physical attributes on the principal soil functions, influenced by the management systems of those soils. Only the agro-forestry systems employing eucalyptus with rice and eucalyptus with soybeans provided improved soil quality when using the two systems analyzed.
\end{abstract}

Key words: Eucalyptus. Soil-quality. Sustainability.

\footnotetext{
*Autor para correspondência

${ }^{1}$ Recebido para publicação em 06/06/2010; aprovado em 29/12/2011

Parte da Dissertação do primeiro autor apresentada ao Programa de Pós-graduação em Ciência do Solo da Universidade Federal de Lavras ${ }^{2}$ Departamento de Ciência do Solo, Universidade Federal de Lavras, Lavras-MG, Brasil, 37.200-000, diego_ufla@yahoo.com.br ${ }^{3}$ Departamento de Ciência do Solo, Universidade Federal de Lavras, Lavras-MG, Brasil, 37.200-000, marx@dcs.ufla.br; niltcuri@dcs.ufla.br ${ }^{4}$ Embrapa Pantanal, Caixa Postal 109, Corumbá-MS, Brasil, 79.320-900, evaldo@ cpap.embrapa.br
} 


\section{INTRODUÇÃO}

No Brasil a expansão de florestas homogêneas com eucalipto em larga escala tem ocupado grandes áreas, sendo que o cerrado tornou-se uma área de importância estratégica para a intensificação das atividades agrossilvopastoris e de produção de madeiras, fibras e energia (FERREIRA et al., 2007). As atividades antrópicas têm gerado degradação ambiental, incluindo erosão e contaminação de solos, sedimentos e corpos d'água, sendo que o modelo impactante atualmente adotado coloca em risco a sustentabilidade dos sistemas produtivos (MOTA; VALLADARES, 2011). Devido o solo ser um importante componente relacionado à produção de madeira, a conservação ou a melhoria da sua qualidade é vital para a sustentação dessa atividade produtiva (CHAER; TÓTOLA, 2007). Nos últimos anos a preocupação com a qualidade do solo tem crescido, na medida em que seu uso e mobilização intensiva podem diminuir a sua capacidade de manter uma produção biológica sustentável (CARVALHO et al., 2004). Assim, é recomendável o monitoramento dos solos sob diferentes sistemas de manejo com vista à preservação da sua qualidade para que o mesmo possa proporcionar uma produção continuada (FIALHO et al., 2008).

Para Doran e Parkin (1994), a qualidade do solo é a capacidade do mesmo funcionar dentro dos limites de um ecossistema natural ou manejado de forma a sustentar a produtividade biológica, manter ou aumentar a qualidade ambiental e promover a saúde das plantas, animais e dos homens. Embora existam vários métodos para monitorar e avaliar a qualidade da água e do ar, nenhum método isolado tem sido amplamente aceito para atribuir um índice de qualidade ao solo, devido à complexidade e variabilidade desse sistema (CONCEIÇÃO et al., 2005; GLOVER et al., 2000).

Conforme Melloni et al. (2008), o desafio dos estudos sobre sustentabilidade está relacionado ao desenvolvimento de métodos para avaliação da qualidade do solo e do ambiente sob a interferência do homem. Atualmente, há um esforço multidisciplinar tentando quantificar diferentes atributos que estão relacionados com a sustentabilidade, traduzindoos na forma de indicadores de qualidade do solo. Diversos trabalhos têm sido conduzidos com o objetivo de identificar os atributos indicadores da qualidade do solo em sistemas agropecuários implantados em substituição aos ecossistemas nativos (CARDOSO et al., 2009; CARDOSO et al., 2011; NEVES et al., 2007; SILVEIRA et al., 2006), e avaliar o efeito integrado dos mesmos, expressos por meio de índices (ARAÚJO et al., 2007; CARDOSO, 2008; MELO FILHO et al., 2007; MELO FILHO et al., 2009). Dois diferentes enfoques têm sido propostos para estabelecer critérios de referência da qualidade do solo: aquele relacionado à área sob vegetação nativa, por representar as condições ecológicas de estabilidade do ambiente; e aquele relacionado a parâmetros agronômicos que maximizem a produção e conservem o meio ambiente (TÓTOLA; CHAER, 2002).

Segundo Vezzani e Mielniczuk (2009), a identificação ou avaliação de um indicador não constitui dificuldade em relação à qualidade do solo, mas sim o planejamento de agroecossistemas complexos que privilegiem o cultivo diversificado de plantas. A complexidade dos ecossistemas é o que faz a diferença para o desempenho eficiente das funções do sistema solo, determinando sua qualidade ambiental, sendo que essa complexidade é alcançada pelo cultivo contínuo e diversificado de plantas. Porém, estudos relacionados à qualidade do solo em áreas florestadas com eucalipto sob diferentes sistemas de manejo são escassos na literatura.

Assim, o objetivo deste trabalho foi estabelecer índices de qualidade do solo sob diferentes sistemas de uso e manejo florestal e cerrado nativo adjacente por meio da avaliação integrada de atributos físicos e químicos dos solos.

\section{MATERIAL E MÉTODOS}

Para a realização deste estudo foi construído um banco de dados de atributos físicos e químicos de Latossolos sob diferentes sistemas de manejo florestal e sob cerrado nativo adjacente, localizados em distintas regiões de Minas Gerais, conforme descrito na Tabela 1. O banco de dados em questão foi elaborado por meio da seleção de informações e dados constantes no arquivo do Setor de Conservação do Solo e da Água, do Departamento de Ciência do Solo da UFLA (Tabelas 2 e 3).

A porosidade do solo e a condutividade hidráulica saturada foram determinados conforme Danielson e Sutherland (1986) e Lima et al. (1990), respectivamente. A densidade do solo, diâmetro médio geométrico dos agregados, pH em água, carbono orgânico total, fósforo, disponível, cátions trocáveis $\left(\mathrm{Ca}^{2+}, \mathrm{Mg}^{2+}, \mathrm{K}^{+}\right)$, alumínio $\left(\mathrm{Al}^{3+}\right)$ e acidez $\left(\mathrm{H}^{+}+\mathrm{Al}^{3+}\right)$ extraíveis foram determinados de acordo com o Manual de Métodos de Análise de Solo (EMPRESA BRASILEIRA DE PESQUISA AGROPECUÁRIA, 1997). A partir destes resultados foram calculados a soma de bases trocáveis (SB) e a saturação por alumínio (m).

A avaliação da qualidade do solo foi realizada a partir do desenvolvimento de dois índices (IQS e IQS $_{2}$ ) que melhor representam as características dos Latossolos sob cerrado, sendo que o IQS foi baseado no modelo proposto para a determinação do índice de deterioração do solo (ISLAM; WEIL, 2000) e o IQS 2 realizado conforme modelo proposto por Karlen e Stott (1994).

Conforme Araújo et al. (2007), para aplicação do IQS $_{1}$ algumas premissas básicas devem ser admitidas: 
Tabela 1 - Caracterização dos sistemas florestais de estudo

\begin{tabular}{|c|c|c|c|c|}
\hline Sistema & Solo & Símbolo & Descrição & Histórico de uso \\
\hline \multicolumn{5}{|c|}{ Campos das Vertentes - MG } \\
\hline Cerrado nativo & $\mathrm{LVA}_{1}$ & CN1 & \multicolumn{2}{|c|}{ Condição original, referencial de sistema em equilíbrio } \\
\hline Eucalipto convencional & $\mathrm{LVA}_{1}$ & EC1 & $\begin{array}{l}\text { Cultivo convencional com eucalipto, sem } \\
\text { aplicação de práticas de manejo após o } \\
\text { plantio }\end{array}$ & $\begin{array}{l}\text { Eucalipto cultivado nos } 13 \text { anos } \\
\text { anteriores à amostragem de solo }\end{array}$ \\
\hline \multicolumn{5}{|c|}{ Centro Leste - Belo Oriente - MG } \\
\hline Cerrado nativo & $\mathrm{LVA}_{2}$ & $\mathrm{CN} 2$ & \multicolumn{2}{|c|}{ Condição original, referencial de sistema em equilíbrio } \\
\hline Eucalipto em nível & $\mathrm{LVA}_{2}$ & EN1 & $\begin{array}{l}\text { Linha de plantio do eucalipto foi } \\
\text { perpendicular ao sentido do declive }\end{array}$ & \multirow{3}{*}{$\begin{array}{l}\text { Eucalipto cultivado nos } 24 \text { anos anteriores } \\
\text { à amostragem de solo. A correção química } \\
\text { de plantio consistiu da aplicação de } 0,09 \\
\text { kg de NPK 6-30-6; } 0,44 \mathrm{~kg} \text { de fosfato } \\
\text { reativo; } 1 \mathrm{~kg} \text { de calcário e } 0,3 \mathrm{~kg} \text { de } \\
\text { cloreto de potássio por planta }\end{array}$} \\
\hline $\begin{array}{l}\text { Eucalipto convencional com } \\
\text { queima de restos culturais }\end{array}$ & $\mathrm{LVA}_{2}$ & $\mathrm{ECq}$ & $\begin{array}{l}\text { Eucalipto cultivado no sentido de } \\
\text { declividade do terreno, sendo realizada a } \\
\text { queima de restos culturais }\end{array}$ & \\
\hline Eucalipto convencional & $\mathrm{LVA}_{2}$ & $\mathrm{EC} 2$ & $\begin{array}{l}\text { Eucalipto cultivado no sentido da } \\
\text { declividade do terreno, sendo os restos } \\
\text { culturais mantidos na superfície }\end{array}$ & \\
\hline \multicolumn{5}{|c|}{ Centro Leste - Guanhães - MG } \\
\hline Cerrado nativo & $\mathrm{LV}_{1}$ & $\mathrm{CN} 3$ & \multicolumn{2}{|c|}{ Condição original, referencial de sistema em equilíbrio } \\
\hline $\begin{array}{l}\text { Eucalipto no sentido da } \\
\text { declividade com queima de } \\
\text { restos culturais }\end{array}$ & $\mathrm{LV}_{1}$ & $\mathrm{EDq}$ & $\begin{array}{l}\text { Eucalipto cultivado no sentido de } \\
\text { declividade do terreno, sendo os restos } \\
\text { culturais queimados }\end{array}$ & $\begin{array}{l}\text { Eucalipto cultivado nos } 28 \text { anos } \\
\text { anteriores à amostragem de solo. A }\end{array}$ \\
\hline $\begin{array}{l}\text { Eucalipto no sentido } \text { da } \\
\text { declividade sem } \\
\text { restos culturais }\end{array}$ & $\mathrm{LV}_{1}$ & ED & $\begin{array}{l}\text { Eucalipto cultivado no sentido de } \\
\text { declividade do terreno, mantendo os } \\
\text { restos culturais na superfície do solo }\end{array}$ & $\begin{array}{l}\text { correção química do solo consistiu da } \\
\text { aplicação durante o plantio } 0,09 \mathrm{~kg} \text { de } \\
\text { NPK 6-30-6; 0,4 kg de fosfato reativo; }\end{array}$ \\
\hline Eucalipto em nível & $\mathrm{LV}_{1}$ & $\mathrm{EN} 2$ & $\begin{array}{l}\text { Linha de plantio do eucalipto é } \\
\text { perpendicular ao sentido do declive }\end{array}$ & $\begin{array}{l}0,35 \mathrm{~kg} \text { de cloreto de potássio e } 1,5 \% \text { de } \\
\text { boro por planta }\end{array}$ \\
\hline \multicolumn{5}{|c|}{ Noroeste - MG } \\
\hline Cerrado nativo & $\mathrm{LV}_{2}$ & $\mathrm{CN} 4$ & Condição original, refere & de sistema em equilíbrio \\
\hline Eucalipto + arroz & $\mathrm{LV}_{2}$ & EA & $\begin{array}{l}\text { Início das atividades do sistema } \\
\text { agrossilvopastoril. Eucalipto consorciado } \\
\text { com arroz, sendo } 4 \text { meses a idade do } \\
\text { eucalipto }\end{array}$ & $\begin{array}{l}\text { No plantio do arroz foram aplicados } 4 \\
\mathrm{t} \text { ha-1 de calcário e } 200 \mathrm{~kg} \mathrm{ha}^{-1} \text { de NPK } \\
5-25-15 \text { e no eucalipto } 240 \mathrm{~kg} \mathrm{ha}^{-1} \text { de } \\
\text { fosfato natural; } 120 \mathrm{~kg} \mathrm{ha}^{-1} \text { de gesso } \\
\text { agrícola, } 48 \mathrm{~kg} \mathrm{ha}^{-1} \text { de óxido de magnésio } \\
\text { e } 37,5 \mathrm{~kg} \mathrm{ha}^{-1} \text { de NPK } 10-28-6\end{array}$ \\
\hline Eucalipto + soja & $\mathrm{LV}_{2}$ & ES & $\begin{array}{l}\text { Eucalipto consorciado com soja, sendo } \\
16 \text { meses a idade do eucalipto }\end{array}$ & $\begin{array}{l}\text { No plantio da soja foram aplicados } 3 \mathrm{t} \\
\mathrm{ha}^{-1} \text { de calcário e } 300 \mathrm{~kg} \mathrm{ha}^{-1} \text { de NPK } \\
2-20-20 \text {. }\end{array}$ \\
\hline Eucalipto + pastagem & $\mathrm{LV}_{2}$ & EP & $\begin{array}{l}\text { Eucalipto consorciado com pastagem } \\
\text { plantada, sendo } 40 \text { meses a idade do } \\
\text { eucalipto }\end{array}$ & $\begin{array}{l}\text { Para a formação da pastagem foi } \\
\text { aplicado } 1 \mathrm{t} \mathrm{ha}^{-1} \text { de calcário }\end{array}$ \\
\hline Eucalipto + pastagem + gado & $\mathrm{LV}_{2}$ & EPG & $\begin{array}{l}\text { Eucalipto consorciado com pastagem } \\
\text { plantada, sendo } 88 \text { meses a idade do } \\
\text { eucalipto }\end{array}$ & $\begin{array}{l}\text { A cada três anos após a implantação das } \\
\text { pastagens foram aplicados } 1 \mathrm{t} \mathrm{ha}^{-1} \text { de } \\
\text { calcário, } 300 \mathrm{~kg} \mathrm{ha}^{-1} \text { de fosfato natural, } \\
200 \mathrm{~kg} \mathrm{ha}^{-1} \text { de superfosfato simples e } 80 \\
\mathrm{~kg} \mathrm{ha}^{-1} \text { de cloreto de potássio }\end{array}$ \\
\hline Eucalipto convencional & $\mathrm{LV}_{2}$ & EC3 & Eucalipto convencional (espaçamento 3x2) & $\begin{array}{l}\text { Aplicação de } 240 \mathrm{~kg} \mathrm{ha}^{-1} \text { de fosfato } \\
\text { natural; } 120 \mathrm{~kg} \mathrm{ha}^{-1} \text { de gesso agrícola, } \\
48 \mathrm{~kg} \mathrm{ha}^{-1} \text { de óxido de magnésio e } \\
37,5 \mathrm{~kg} \mathrm{ha}^{-1} \text { de NPK } 10-28-6\end{array}$ \\
\hline
\end{tabular}

LVA: Latossolo Vermelho-Amarelo; LV: Latossolo Vermelho 
Tabela 2 - Densidade do solo, porosidade total, macro e microporosidade, condutividade hidráulica saturada (ks) e diâmetro médio geométrico (DMG) dos agregados para os diferentes sistemas de uso e manejo do eucalipto

\begin{tabular}{|c|c|c|c|c|c|c|c|}
\hline \multirow{2}{*}{ Uso e manejo do solo } & \multirow{2}{*}{ Solo } & Densidade do solo & Porosidade total & Macro & Micro & ks & DMG \\
\hline & & ---- $\mathrm{kg} \mathrm{dm}^{-3}$---- & \multicolumn{3}{|c|}{------------- $\mathrm{m}^{3} \mathrm{~m}^{-3}$------------ } & $-\mathrm{mm} \mathrm{h}^{-1}-$ & $-\mathrm{mm}-$ \\
\hline \multicolumn{8}{|c|}{ Campos das Vertentes - MG } \\
\hline $\mathrm{CN} 1$ & $\mathrm{LVA}_{1}$ & 1,11 & 0,55 & 0,18 & 0,37 & 38,0 & 4,87 \\
\hline EC1 & $\mathrm{LVA}_{1}$ & 1,15 & 0,53 & 0,07 & 0,46 & 41,3 & 4,77 \\
\hline \multicolumn{8}{|c|}{ Centro Leste - Belo Oriente - MG } \\
\hline $\mathrm{CN} 2$ & $\mathrm{LVA}_{2}$ & 0,93 & 0,60 & 0,30 & 0,30 & 191,0 & 4,55 \\
\hline EN1 & $\mathrm{LVA}_{2}$ & 1,13 & 0,55 & 0,26 & 0,29 & 180,0 & 4,57 \\
\hline $\mathrm{ECq}$ & $\mathrm{LVA}_{2}$ & 1,21 & 0,51 & 0,19 & 0,32 & 161,0 & 4,46 \\
\hline $\mathrm{EC} 2$ & $\mathrm{LVA}_{2}$ & 1,19 & 0,52 & 0,21 & 0,3 & 152,0 & 4,35 \\
\hline \multicolumn{8}{|c|}{ Centro Leste - Guanhães - MG } \\
\hline $\mathrm{CN} 3$ & $\mathrm{LV}_{1}$ & 0,87 & 0,65 & 0,33 & 0,32 & 230,0 & 4,90 \\
\hline EDq & $\mathrm{LV}_{1}$ & 1,14 & 0,54 & 0,17 & 0,37 & 174,0 & 4,92 \\
\hline ED & $\mathrm{LV}_{1}$ & 1,13 & 0,55 & 0,18 & 0,37 & 160,0 & 4,92 \\
\hline EN2 & $\mathrm{LV}_{1}$ & 1,18 & 0,54 & 0,17 & 0,37 & 75,0 & 4,89 \\
\hline \multicolumn{8}{|c|}{ Noroeste - MG } \\
\hline $\mathrm{CN} 4$ & $\mathrm{LV}_{2}$ & 1,07 & 0,54 & 0,24 & 0,30 & 733,7 & 4,39 \\
\hline EA & $\mathrm{LV}_{2}$ & 1,06 & 0,54 & 0,23 & 0,31 & 136,4 & 4,05 \\
\hline ES & $\mathrm{LV}_{2}$ & 1,01 & 0,55 & 0,19 & 0,36 & 348,6 & 4,17 \\
\hline EP & $\mathrm{LV}_{2}$ & 1,15 & 0,54 & 0,23 & 0,31 & 74,95 & 3,92 \\
\hline EPG & $\mathrm{LV}_{2}$ & 1,13 & 0,56 & 0,27 & 0,29 & 128,6 & 4,00 \\
\hline EC3 & $\mathrm{LV}_{2}$ & 1,14 & 0,52 & 0,28 & 0,24 & 214,5 & 4,33 \\
\hline
\end{tabular}

CN1, CN2, CN3 e CN4: cerrado nativo; EC1, EC2 e EC3: eucalipto sob cultivo convencional; EDq: eucalipto plantado no sentido da declividade com queima de restos culturais; ED: eucalipto plantado no sentido da declividade sem queima de restos culturais; EN1 e EN2: eucalipto plantado em nível; EA: eucalipto + arroz; ES: eucalipto + soja; EP: eucalipto + pastagem; EPG: eucalipto + pastagem + gado; LVA: Latossolo Vermelho-Amarelo; LV: Latossolo Vermelho

os ecossistemas naturais, caracterizados pelo mínimo de intervenção antrópica e de esperado equilíbrio, devem ser considerados comoreferência; as categorias de atributos físicos e químicos de qualidade do solo contribuem equitativamente para a qualidade do solo, sendo atribuído a cada categoria o mesmo peso ponderado; e os indicadores dentro de cada categoria de atributos têm a mesma importância relativa.

As diferenças entre os atributos do solo dos sistemas de uso e manejo, comparadas à linha base dos atributos do solo das respectivas áreas de referência foram calculadas e expressas como as médias dos desvios dos valores individuais de cada atributo. A média geral dos desvios de cada atributo do solo representa a sua deterioração em relação à referência. Para os ecossistemas considerados como referência, $\mathrm{CN} 1$ e CN2 para os Latossolos Vermelho-Amarelos e CN3 e CN4 para os Latossolos Vermelhos, foi atribuído o valor 1,0 para a qualidade do solo e sua diferença em relação à deterioração do solo nos sistemas florestais constitui o índice de qualidade do solo $\left(\mathrm{IQS}_{1}\right)$. O cálculo do $\mathrm{IQS}_{1}$ processou-se em duas etapas, conforme as equações 1 e 2 .

$Q_{\Lambda}=\frac{\left(\frac{w 1-k 1}{k 1}\right)+\left(\frac{w 2-k 2}{k 2}\right)+\left(\frac{w 3-k 3}{k 3}\right)+\left(\frac{w n-k n}{k n}\right)}{n}$

$$
I Q S_{1}=1-\left(\frac{Q a q+Q a f}{2}\right)
$$

Em que: QA - refere-se à média dos desvios dos indicadores de cada atributo em relação à referência; w - refere-se ao valor do indicador medido nos sistemas em estudo; $\mathrm{k}$ - refere-se ao valor do indicador medido no sistema referência; $\mathrm{n}$ - é o número de indicadores que compõem cada conjunto de atributos; Qaq - é a média dos desvios dos atributos químicos do solo; e Qaf - é a média dos desvios dos atributos físicos do solo. 
Tabela 3 - Valores de $\mathrm{pH}$, cálcio trocável $\left(\mathrm{Ca}^{2+}\right)$, magnésio trocável $\left(\mathrm{Mg}^{2+}\right)$, soma de bases $(\mathrm{SB})$, capacidade de troca catiônica efetiva $(\mathrm{t})$, potássio disponível (K), fósforo disponível (P), saturação por alumínio (m), matéria orgânica do solo (MOS), carbono orgânico total (COT) e estoque de carbono para os diferentes sistemas de manejo do eucalipto

\begin{tabular}{|c|c|c|c|c|c|c|c|c|c|c|c|c|}
\hline \multirow{2}{*}{ Uso e manejo do solo } & \multirow{2}{*}{ Solo } & $\mathrm{pH}$ & $\mathrm{Ca}^{2+}$ & $\mathrm{Mg}^{2+}$ & SB & $\mathrm{t}$ & $\mathrm{K}$ & $\mathrm{P}$ & $\mathrm{m}$ & MOS & COT & Estoque de carbono \\
\hline & & $\mathrm{H}_{2} \mathrm{O}$ & \multicolumn{4}{|c|}{$\mathrm{cmol}_{\mathrm{c}} \mathrm{dm}^{-3}$} & \multicolumn{2}{|c|}{$\mathrm{mg} \mathrm{dm}{ }^{-3}$} & $\%$ & \multicolumn{2}{|c|}{$\mathrm{g} \mathrm{kg}^{-1}$} & $\mathrm{Mg} \mathrm{ha}^{-1}$ \\
\hline \multicolumn{13}{|c|}{ Campo das Vertentes - MG } \\
\hline $\mathrm{CN} 1$ & $\mathrm{LVA}_{1}$ & 5,15 & 0,55 & 0,15 & 0,75 & 1,05 & 17,00 & 1,75 & 30,3 & 46,83 & 27,17 & 59,32 \\
\hline EC1 & $\mathrm{LVA}_{1}$ & 5,15 & 0,95 & 0,15 & 1,10 & 1,65 & 15,00 & 2,75 & 49,0 & 40,02 & 23,22 & 50,75 \\
\hline \multicolumn{13}{|c|}{ Centro Leste - Belo Oriente - MG } \\
\hline $\mathrm{CN} 2$ & $\mathrm{LVA}_{2}$ & 4,60 & 0,76 & 0,46 & 1,36 & 1,58 & 56,44 & 0,88 & 9,3 & 64,13 & 37,20 & 69,2 \\
\hline EN1 & $\mathrm{LVA}_{2}$ & 4,60 & 0,46 & 0,28 & 0,83 & 0,96 & 35,35 & 0,54 & 5,7 & 58,44 & 33,90 & 88,6 \\
\hline $\mathrm{ECq}$ & $\mathrm{LVA}_{2}$ & 4,80 & 0,61 & 0,37 & 1,10 & 1,28 & 47,13 & 0,72 & 7,6 & 47,58 & 27,60 & 65,7 \\
\hline $\mathrm{EC} 2$ & $\mathrm{LVA}_{2}$ & 5,10 & 0,56 & 0,34 & 1,01 & 1,17 & 44,02 & 0,67 & 7,0 & 63,10 & 36,60 & 76,6 \\
\hline \multicolumn{13}{|c|}{ Centro Leste - Guanhães - MG } \\
\hline $\mathrm{CN} 3$ & $\mathrm{LV}_{1}$ & 4,33 & 0,51 & 0,18 & 0,84 & 2,63 & 56,50 & 3,08 & 68,1 & 63,20 & 36,69 & 63,84 \\
\hline EDq & $\mathrm{LV}_{1}$ & 4,66 & 1,54 & 0,34 & 1,98 & 2,99 & 37,17 & 3,74 & 44,1 & 44,40 & 25,79 & 58,80 \\
\hline ED & $\mathrm{LV}_{1}$ & 4,37 & 0,92 & 0,35 & 1,41 & 2,91 & 51,17 & 3,83 & 55,6 & 58,10 & 33,70 & 76,16 \\
\hline EN2 & $\mathrm{LV}_{1}$ & 4,44 & 0,93 & 0,27 & 1,30 & 2,83 & 40,00 & 3,11 & 64,3 & 54,90 & 31,86 & 75,19 \\
\hline \multicolumn{13}{|c|}{ Noroeste - MG } \\
\hline $\mathrm{CN} 4$ & $\mathrm{LV}_{2}$ & 4,88 & 0,44 & 0,17 & 0,76 & 2,13 & 58,92 & 0,65 & 64,5 & 29,39 & 17,05 & 34,91 \\
\hline EA & $\mathrm{LV}_{2}$ & 4,98 & 0,93 & 0,51 & 1,53 & 1,75 & 28,67 & 0,32 & 27,0 & 20,56 & 11,93 & 24,88 \\
\hline ES & $\mathrm{LV}_{2}$ & 5,18 & 1,15 & 0,76 & 2,01 & 1,29 & 35,83 & 0,54 & 33,5 & 21,55 & 12,50 & 24,87 \\
\hline $\mathrm{EP}$ & $\mathrm{LV}_{2}$ & 5,20 & 1,02 & 0,68 & 1,79 & 2,35 & 27,92 & 2,66 & 33,0 & 22,54 & 13,08 & 29,11 \\
\hline EPG & $\mathrm{LV}_{2}$ & 5,28 & 0,95 & 0,50 & 1,50 & 2,13 & 23,33 & 0,30 & 32,0 & 20,00 & 11,60 & 26,58 \\
\hline EC3 & $\mathrm{LV}_{2}$ & 4,98 & 0,57 & 0,20 & 0,88 & 2,28 & 37,58 & 1,35 & 62,5 & 25,69 & 14,90 & 34,37 \\
\hline
\end{tabular}

CN1, CN2, CN3 e CN4: cerrado nativo; EC1, EC2 e EC3: eucalipto sob cultivo convencional; EDq: eucalipto plantado no sentido da declividade com queima de restos culturais; ED: eucalipto plantado no sentido da declividade sem queima de restos culturais; EN1 e EN2: eucalipto plantado em nível; EA: eucalipto + arroz; ES: eucalipto + soja; EP: eucalipto + pastagem; EPG: eucalipto + pastagem + gado; LVA: Latossolo Vermelho-Amarelo; LV: Latossolo Vermelho

$\mathrm{OIQS}_{2}$ foi gerado a partir de um modelo aditivo que considera as funções principais do solo e os indicadores de qualidade a elas associados, sendo atribuídos pesos para as funções e para os indicadores (KARLEN; STOTT, 1994). O IQS foi aplicado por Melo Filho et al. (2007) e Cardoso (2008), seu cálculo processou-se em duas etapas, conforme as equações 3 e 4 .

$\mathrm{Q}_{\mathrm{FPn}}=\mathrm{I}_{1}\left(\mathrm{~W}_{1}\right)+\mathrm{I}_{2}\left(\mathrm{~W}_{2}\right)+\mathrm{In}\left(\mathrm{W}_{\mathrm{n}}\right)$

$\mathrm{IQS}_{2}=\mathrm{Q}_{\mathrm{FP} 1}\left(\mathrm{~W}_{\mathrm{FP} 1}\right)+\mathrm{Q}_{\mathrm{FP} 2}\left(\mathrm{~W}_{\mathrm{FP} 2}\right)+\mathrm{Q}_{\mathrm{FP} 3}\left(\mathrm{~W}_{\mathrm{FP} 3}\right)+\mathrm{Q}_{\mathrm{FPn}}\left(\mathrm{W}_{\mathrm{FPn}}\right)$

em que: QFPn - refere-se à qualidade da função principal do solo; I - refere-se aos escores padronizados dos indicadores de qualidade relacionados a cada função principal; w - refere-se aos ponderadores relacionados a cada indicador ou a cada função principal; e IQS - é o índice integrado da qualidade do solo.
Quatro funções do solo relacionadas com a sustentabilidade do sistema foram definidas neste estudo para a obtenção do $\mathrm{IQS}_{2}$ : receber, armazenar e suprir água; promover o crescimento das raízes; armazenar, suprir e ciclar nutrientes; e promover a conservação do solo. Para cada função do solo foi assumida a igualdade de importância, com atribuição de peso 0,25 para cada uma delas (Tabela 4). Os pesos numéricos foram atribuídos às funções de acordo com o grau de importância da mesma para o funcionamento do solo e no desempenho da função para a qual o índice está sendo calculado.

Segundo esta metodologia, o somatório dos pesos de todas as funções principais deve resultar no valor 1,0 (um), sendo este o valor do IQS para um solo considerado ideal em relação ao objetivo considerado. Após atribuir os pesos relativos para as funções, foram identificados e priorizados os indicadores e ponderadores que influenciam cada uma, em diversos graus, sendo o somatório geral dos pesos dos indicadores em cada nível igual a 1,0 (Tabela 4). 
Tabela 4 - Funções principais, indicadores de qualidade e índice de qualidade do solo (IQS $\left.{ }_{2}\right)$

\begin{tabular}{|c|c|c|c|c|c|c|}
\hline \multirow{2}{*}{ Funções principais } & \multirow{2}{*}{$\begin{array}{c}\text { Ponderadores } \\
\text { das funções }\end{array}$} & \multirow{2}{*}{$\begin{array}{l}\text { Indicadores } \\
\text { de qualidade }^{1}\end{array}$} & \multirow{2}{*}{$\begin{array}{l}\text { Ponderadores } \\
\text { dos indicadores }\end{array}$} & \multicolumn{2}{|c|}{ Limite críticos } & \multirow{2}{*}{$\begin{array}{c}\text { Referências dos } \\
\text { limites críticos }\end{array}$} \\
\hline & & & & Inferior & Superior & \\
\hline \multirow{5}{*}{ Receber, armazenar e suprir água } & \multirow{5}{*}{0,25} & $\mathrm{RP}$ & 0,15 & 1,70 & & $\begin{array}{l}\text { Grant e Lanfond } \\
\text { (1993) }\end{array}$ \\
\hline & & Macro & 0,15 & 0,10 & 0,30 & Cardoso (2008) \\
\hline & & Ds & 0,15 & 1,20 & & Freitas (2010) \\
\hline & & MOS & 0,40 & 4,00 & & Freitas (2010) \\
\hline & & $\mathrm{Pt}$ & 0,15 & 0,36 & 0,55 & Cardoso (2008) \\
\hline \multirow{5}{*}{ Promover o crescimento de raízes } & \multirow{5}{*}{0,25} & MOS & 0,40 & 4,00 & & Freitas (2010) \\
\hline & & Ds & 0,15 & 1,20 & & Freitas (2010) \\
\hline & & $\mathrm{RP}$ & 0,15 & 1,70 & & $\begin{array}{l}\text { Grant e Lanfond } \\
\text { (1993) }\end{array}$ \\
\hline & & SB & 0,15 & 1,20 & & Freitas (2010) \\
\hline & & $\mathrm{m}$ & 0,15 & 50 & & Lepsch (1983) \\
\hline \multirow{4}{*}{ Armazenar, suprir e ciclar nutrientes } & \multirow{4}{*}{0,25} & MOS & 0,40 & 4,00 & & Freitas (2010) \\
\hline & & SB & 0,30 & 1,20 & & Freitas (2010) \\
\hline & & $\mathrm{m}$ & 0,15 & 50 & & Lepsch (1983) \\
\hline & & $\mathrm{pH}$ & 0,15 & 5,00 & 6,50 & Freitas (2010) \\
\hline \multirow{6}{*}{ Promover a conservação do solo } & \multirow{6}{*}{0,25} & DMG & 0,25 & 2,50 & & $\begin{array}{l}\text { Alvarenga et al. } \\
(1986)\end{array}$ \\
\hline & & ks & 0,25 & 127 & & Freitas (2010) \\
\hline & & MOS & 0,15 & 4,00 & & Freitas (2010) \\
\hline & & $\mathrm{Pt}$ & 0,10 & 0,36 & 0,55 & Cardoso (2008) \\
\hline & & Macro & 0,10 & 0,10 & 0,30 & Cardoso (2008) \\
\hline & & Ds & 0,15 & 1,20 & & Freitas (2010) \\
\hline
\end{tabular}

${ }^{1} \mathrm{Rp}$ - resistência do solo à penetração; Macro - macroporosidade; Ds - densidade do solo; MOS - matéria orgânica do solo; Pt - porosidade total; SB - soma de bases; $m$ - saturação por alumínio; DMG - diâmetro médio geométrico dos agregados; Ks - permeabilidade do solo saturado

Os indicadores de qualidade do solo por possuírem diferentes unidades de medida foram padronizados para escores que variam de 0 a 1 , aplicando-se a função de padronização de escores, desenvolvida por Wymore (1993), conforme equação 5.

$\mathrm{v}=\frac{1}{1+((\mathrm{B}-\mathrm{L}) /(\mathrm{x}-\mathrm{L}))^{2 \mathrm{~S}(\mathrm{~B}+\mathrm{x}-2 \mathrm{~L})}}$

em que: v - é a pontuação padronizada; B - é o valor crítico ou limite-base do indicador, cuja pontuação equivale a 0,5; L - é o limite inferior ou o pior valor do indicador, podendo ser zero; $\mathrm{S}$ - é a inclinação da tangente da curva no limite-base ou no valor crítico do indicador; e x - é o valor do indicador medido no campo.

Para aplicar-se a equação de Wymore (1993), inicialmente foi necessário calcular a inclinação (S) da tangente da curva de pontuação no valor crítico do indicador, conforme equação 6 .

$$
\mathrm{S}=\frac{\log \left(\frac{1}{\mathrm{v}}\right)-1}{\log \left(\frac{\mathrm{B}-\mathrm{L}}{\mathrm{x}-\mathrm{L}}\right) * 2(\mathrm{~B}+\mathrm{x}-2 * \mathrm{~L})}
$$

Segundo Karlen e Stott (1994), a curva de padronização do tipo "mais é melhor" possui declividade (S) positiva e é utilizada para padronização de indicadores em que maiores valores apontam para a melhoria da qualidade do solo, a exemplo da soma de bases, CTC efetiva, matéria orgânica, estabilidade de agregados, permeabilidade do solo saturado e carbono orgânico total (Figura 1A); "valor ótimo" possui inclinação positiva e é utilizada para indicadores que apresentam um efeito positivo na qualidade do solo até determinado valor, a partir do qual sua influência é detrimental ou negativa, a exemplo da porosidade total, macroporosidade e $\mathrm{pH}$, (Figura 1B). As 
Figura 1 - Funções de pontuação padronizadas conforme a natureza do indicador de qualidade do solo

A) "mais é melhor"

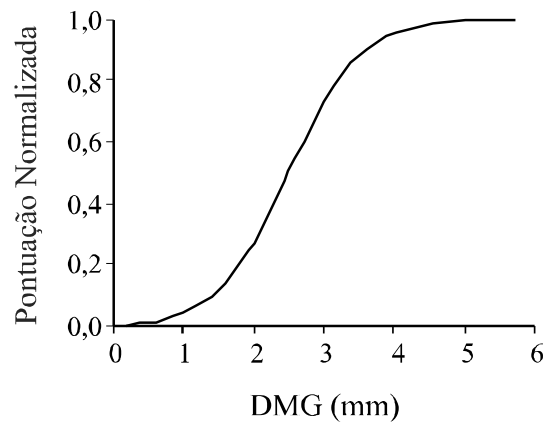

B) "ótimo"

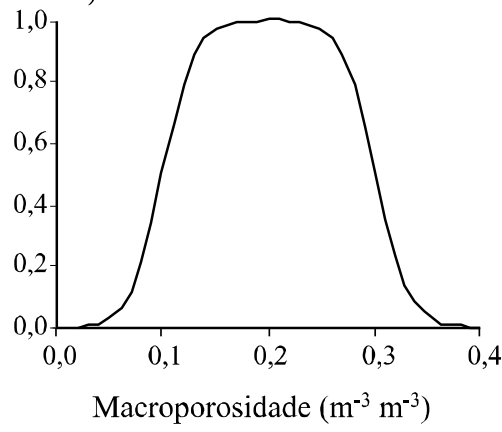

C) "menos é melhor"

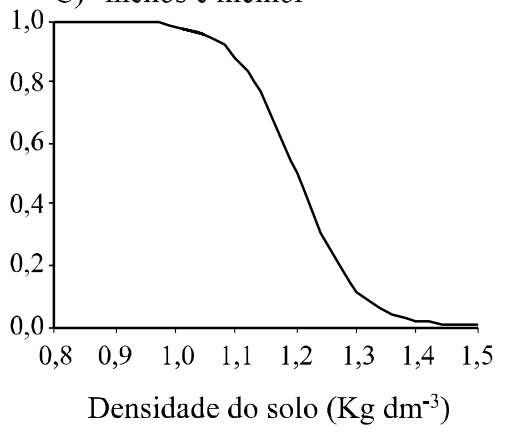

curvas de padronização do tipo "menos é melhor” possuem declividade negativa e padronizam indicadores como a densidade do solo, resistência do solo à penetração e saturação por alumínio, em que a qualidade está associada a menores valores dos mesmos (Figura 1C).

Conforme Melo Filho et al. (2007), na padronização dos atributos são utilizados dois valores-limite dos indicadores de qualidade, o superior e o inferior, e um valor crítico que é aquele no qual a função de pontuação é igual a 0,5 e equivale aos pontos médios entre os valores limites do indicador de qualidade avaliado. Os valores-limite superiores são quando a função de pontuação equivale a 1 , quando o atributo do solo medido está em nível ótimo. Os valores-limite inferiores são aqueles em que a função de pontuação equivale a 0 (zero), quando o atributo do solo está em nível inaceitável. Os valores críticos são aqueles nos quais a função de pontuação é igual a 0,5 .

Os índices de qualidade do solo gerados foram avaliados através da análise de agrupamento de dados, definindo-se o número de grupos pelo critério de Ward. As médias de cada grupo foram comparadas entre si e diferenciadas dos demais pelo teste $\mathrm{t}(p=0,05)$, sendo as análises conduzidas no software estatístico R, utilizandose o pacote cluster (MAECHLER et al., 2005).

\section{RESULTADOS E DISCUSSÃO}

O sistema de eucalipto convencional (EC1) instalado no LVA dos Campos das Vertentes possui sua produção voltada para o setor moveleiro, sendo o manejo manual e sem a introdução de insumos agrícolas. As plantas são conduzidas durante vários anos com pouca interferência humana e o sistema tende a estabilizar-se. Com isto, este sistema apresentou uma pequena deterioração dos atributos físicos (5\%) e químicos (2\%), indicando um IQS muito próximo ao do cerrado nativo da mesma região (Tabela 5).

No $\mathrm{LVA}_{2}$ e $\mathrm{LV}_{1}$ os plantios foram instalados com revolvimento mínimo do solo, sendo realizados em covas feitas manualmente com dimensões de $30 \times 30 \times 30 \mathrm{~cm}$. No $\mathrm{LVA}_{2}$ os atributos químicos e físicos do solo foram deteriorados e apresentaram redução do $\mathrm{IQS}_{1}$ em relação ao sistema nativo (Figura 2A). Os sistemas florestais instalados no Latossolo Vermelho $\left(\mathrm{LV}_{1}\right)$ apresentaram forte deterioração dos atributos físicos do solo (63\%) quando comparados ao sistema nativo (CN3), porém os atributos químicos aumentaram acima de $17 \%$, minimizando as reduções do $\mathrm{IQS}_{1}$ para este solo (Tabela 5). Nesta região, o sistema eucalipto em nível (EN2) apresentou reduzido $\mathrm{IQS}_{1}$, devido à baixa porosidade total e macroporosidade, que influenciaram uma redução de $67 \%$ da permeabilidade do solo à água (Tabela 2) e $29 \%$ do $\mathrm{IQS}_{1}$.

Dentre os sistemas que utilizam a queima dos restos culturais (EDq e ECq), verifica-se que estes não apresentaram diferenças de IQS quando comparados aos sistemas semelhantes da mesma região que não utilizam a queima (ED e EC2, respectivamente) (Figura 2A), pois a queima contribuiu para deteriorar os atributos físicos e melhorar, em pequena magnitude, os atributos químicos. Isto ocorre porque a queima provoca aumento nos teores de $\mathrm{N}$-nitrato, potássio, cálcio + magnésio, $\mathrm{pH}$ e diminuição nos teores de $\mathrm{N}$-amônio e alumínio trocável (RHEINHEIMER et al., 2003).

Os sistemas agrossilvopastoris anteriores à entrada do gado na área (EA, ES, EP) e instalados sobre o Latossolo Vermelho $\left(\mathrm{LV}_{2}\right)$ do Noroeste de Minas Gerais foram os únicos sistemas que apresentaram aumento do $\mathrm{IQS}_{1}$, com valores entre 17 e $23 \%$, em comparação aos sistemas nativos (Tabela 5 e Figura 2A), sendo que os atributos físicos indicadores da qualidade do solo foram deteriorados e os atributos químicos tiveram um forte 
Tabela 5 - Deterioração dos atributos químicos e físicos em relação aos sistemas nativos de referência e índice de qualidade do solo $\left(\mathrm{IQS}_{1}\right.$ ) para diferentes sistemas de uso e manejo de eucalipto

\begin{tabular}{|c|c|c|c|c|}
\hline \multirow{2}{*}{ Sistema de uso e manejo } & \multirow{2}{*}{ Solo } & \multicolumn{2}{|c|}{ Deterioração dos atributos em relação aos sistemas nativos } & \multirow[t]{2}{*}{ IQS } \\
\hline & & Físicos & Químicos & \\
\hline \multicolumn{5}{|c|}{ Campos das Vertentes - MG } \\
\hline CN1 & $\mathrm{LVA}_{1}$ & - & - & 1,00 \\
\hline $\mathrm{EC} 1$ & $\mathrm{LVA}_{1}$ & $-0,051$ & $-0,026$ & 0,99 \\
\hline \multicolumn{5}{|c|}{ Centro Leste - Belo Oriente - MG } \\
\hline $\mathrm{CN} 2$ & $\mathrm{LVA}_{2}$ & - & - & 1,00 \\
\hline EN1 & $\mathrm{LVA}_{2}$ & $-0,214$ & $-0,139$ & 0,82 \\
\hline $\mathrm{ECq}$ & $\mathrm{LVA}_{2}$ & $-0,340$ & $-0,102$ & 0,78 \\
\hline $\mathrm{EC} 2$ & $\mathrm{LVA}_{2}$ & $-0,278$ & $-0,118$ & 0,80 \\
\hline \multicolumn{5}{|c|}{ Centro Leste - Guanhães - MG } \\
\hline CN3 & $\mathrm{LV}_{1}$ & - & - & 1,00 \\
\hline $\mathrm{EDq}$ & $\mathrm{LV}_{1}$ & 0,361 & $-0,720$ & 0,82 \\
\hline ED & $\mathrm{LV}_{1}$ & 0,293 & $-0,635$ & 0,83 \\
\hline EN2 & $\mathrm{LV}_{1}$ & 0,172 & $-0,753$ & 0,71 \\
\hline \multicolumn{5}{|c|}{ Noroeste - MG } \\
\hline $\mathrm{CN} 4$ & $\mathrm{LV}_{2}$ & - & - & 1,00 \\
\hline EA & $\mathrm{LV}_{2}$ & $-0,123$ & 0,333 & 1,17 \\
\hline ES & $\mathrm{LV}_{2}$ & $-0,167$ & 0,573 & 1,23 \\
\hline EP & $\mathrm{LV}_{2}$ & $-0,300$ & 0,562 & 1,18 \\
\hline EPG & $\mathrm{LV}_{2}$ & $-0,362$ & 0,333 & 1,04 \\
\hline EC3 & $\mathrm{LV}_{2}$ & $-0,262$ & 0,002 & 0,92 \\
\hline
\end{tabular}

CN1, CN2, CN3 e CN4: cerrado nativo; EC1, EC2 e EC3: eucalipto sob cultivo convencional; EDq: eucalipto plantado no sentido da declividade com queima de restos culturais; ED: eucalipto plantado no sentido da declividade sem queima de restos culturais; EN1 e EN2: eucalipto plantado em nível; EA: eucalipto + arroz; ES: eucalipto + soja; EP: eucalipto + pastagem; EPG: eucalipto + pastagem + gado; LVA: Latossolo Vermelho-Amarelo; LV: Latossolo Vermelho

Figura 2 - Dendrograma de agrupamento para os sistemas de uso e manejo, calculados pelo critério de Ward, para IQS $\mathrm{I}_{1}(\mathrm{~A})$ e IQS 2 (B)
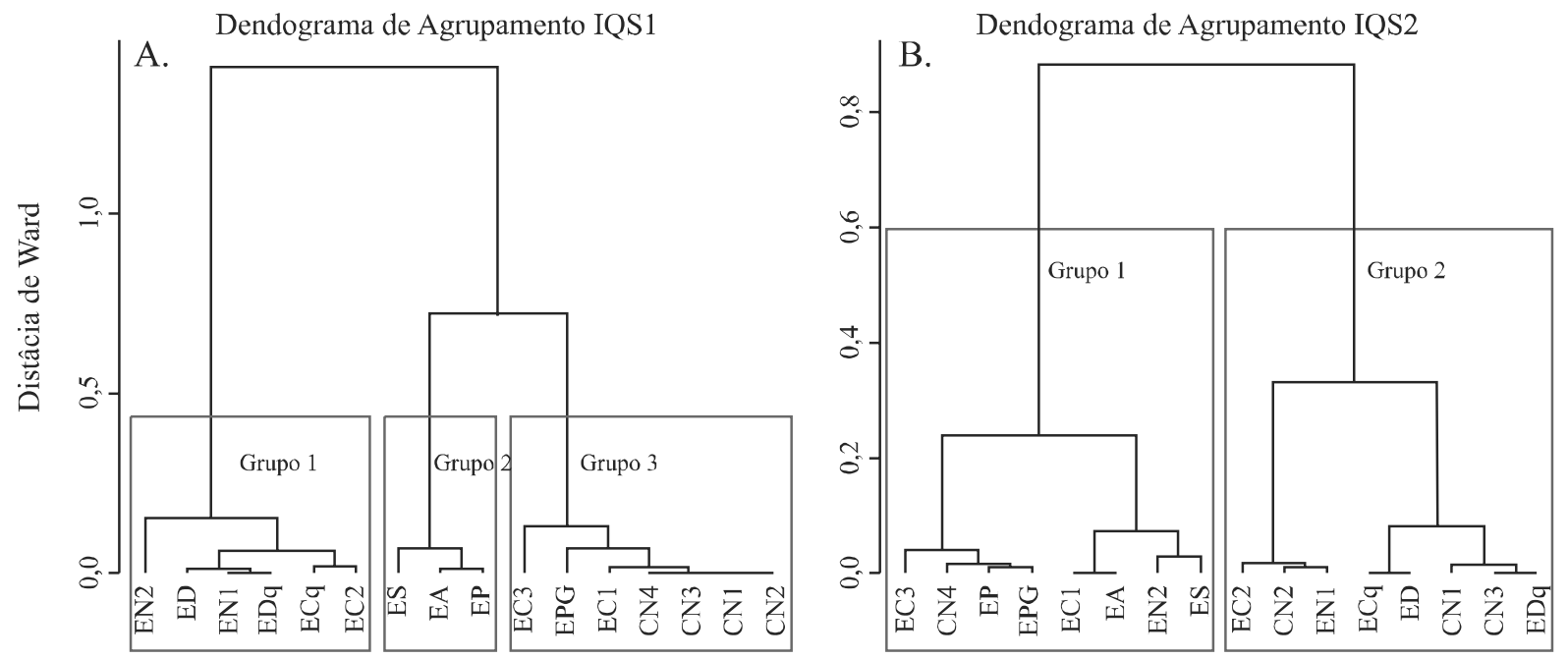

Dose de Superfosfato Simples $\left(\mathrm{kg} \mathrm{m}^{-3}\right)$ 
aumento, devido à constante adição de corretivos de acidez e fertilizantes (Tabela 1). Contudo, estes sistemas não podem ser considerados como excelente opção de manejo e uso dos solos, pois mesmo a parte química tendo melhorado, esta não é capaz de sustentar, isoladamente, a produção agrícola. Segundo Carvalho et al. (2004), os sistemas agrossilvopastoris constituem uma alternativa de produção agropecuária que minimiza o efeito da intervenção humana, pois a consorciação de várias espécies dentro de uma mesma área eleva a diversidade do ecossistema e são aproveitadas as interações benéficas entre as plantas de diferentes ciclos, portes e funções.

Araújo et al. (2007), utilizando o modelo proposto por Islam e Weil (2000), observaram que o florestamento de pinus contribuiu para a melhoria das propriedades físicas do solo, sendo esse resultado possivelmente um reflexo da não mobilização do solo e da manutenção de uma camada de serrapilheira sobre sua superfície. O valor de IQS calculado por estes autores foi de 0,51 , indicando que esse tipo de uso e manejo também pode impactar negativamente a qualidade intrínseca do solo, apesar da melhoria nos seus atributos físicos e da cobertura da superfície do terreno, protegendo o solo da ação erosiva da chuva e do vento.

Cardoso (2008), utilizando a metodologia do $\mathrm{IQS}_{1}$ encontrou valores de 0,$68 ; 0,64 ; 0,62 ; 0,75$ e 0,84 para pastagens cultivadas com 27; 26 e 11 anos de instalação e pastagem nativa com pastejo contínuo e sem pastejo por três anos, respectivamente, para a camada superficial de solos do Pantanal, o que mostra a tendência de redução deste índice em áreas sob pastagens.

Os valores do $\mathrm{IQS}_{2}$, desenvolvidos a partir do estabelecimento das funções do solo e dos indicadores a elas associados, indicaram redução da qualidade do solo nos sistemas estudados (Tabela 6). Na determinação do $\mathrm{IQS}_{2}$ os limites críticos dos indicadores foram estabelecidos buscando o pleno desenvolvimento da função do solo; com isto, foram encontrados baixos valores de qualidade do solo para os todos os sistemas, incluindo os nativos.

Tabela 6 - Funções principais dos solos e índice de qualidade do solo $\left(\mathrm{IQS}_{2}\right)$

\begin{tabular}{|c|c|c|c|c|c|}
\hline \multirow[b]{2}{*}{ Sistema de uso e manejo } & \multicolumn{4}{|c|}{ Funções principais dos solos } & \multirow[b]{2}{*}{$\mathrm{IQS}_{2}$} \\
\hline & $\begin{array}{l}\text { Receber, armazenar } \\
\text { e suprir água }\end{array}$ & $\begin{array}{c}\text { Promover o } \\
\text { crescimento de raízes }\end{array}$ & $\begin{array}{l}\text { Armazenar, suprir } \\
\text { e ciclar nutrientes }\end{array}$ & $\begin{array}{c}\text { Manter a } \\
\text { conservação do solo }\end{array}$ & \\
\hline \multicolumn{6}{|c|}{ Campos das Vertentes - MG - LVA1 } \\
\hline $\mathrm{CN} 1$ & 0,1584 & 0,1510 & 0,1504 & 0,1577 & 0,62 \\
\hline $\mathrm{EC} 1$ & 0,1293 & 0,1331 & 0,1283 & 0,1322 & 0,52 \\
\hline \multicolumn{6}{|c|}{ Centro Leste - Belo Oriente - MG - LVA 2} \\
\hline $\mathrm{CN} 2$ & 0,1555 & 0,1937 & 0,1789 & 0,2050 & 0,73 \\
\hline EN1 & 0,1705 & 0,1684 & 0,1602 & 0,2160 & 0,72 \\
\hline $\mathrm{ECq}$ & 0,1576 & 0,1401 & 0,1534 & 0,2020 & 0,65 \\
\hline EC2 & 0,1825 & 0,1658 & 0,1834 & 0,2087 & 0,74 \\
\hline \multicolumn{6}{|c|}{ Centro Leste - Guanhães - MG - LV } \\
\hline $\mathrm{CN} 3$ & 0,1712 & 0,1811 & 0,1245 & 0,1374 & 0,61 \\
\hline EDq & 0,1517 & 0,1463 & 0,1544 & 0,1551 & 0,61 \\
\hline ED & 0,2046 & 0,1470 & 0,1422 & 0,1587 & 0,65 \\
\hline EN2 & 0,1651 & 0,1263 & 0,1288 & 0,1558 & 0,58 \\
\hline \multicolumn{6}{|c|}{ Noroeste - MG - LV } \\
\hline CN4 & 0,1213 & 0,0778 & 0,0691 & 0,2074 & 0,48 \\
\hline EA & 0,1170 & 0,1162 & 0,1128 & 0,1774 & 0,52 \\
\hline ES & 0,1060 & 0,1143 & 0,1298 & 0,2021 & 0,55 \\
\hline EP & 0,1024 & 0,1038 & 0,1270 & 0,1391 & 0,47 \\
\hline EPG & 0,0870 & 0,1002 & 0,1195 & 0,1567 & 0,46 \\
\hline EC3 & 0,1105 & 0,0653 & 0,0694 & 0,1996 & 0,44 \\
\hline
\end{tabular}

CN1, CN2, CN3 e CN4: cerrado nativo; EC1, EC2 e EC3: eucalipto sob cultivo convencional; EDq: eucalipto plantado no sentido da declividade com queima de restos culturais; ED: eucalipto plantado no sentido da declividade sem queima de restos culturais; EN1 e EN2: eucalipto plantado em nível; EA: eucalipto + arroz; ES: eucalipto + soja; EP: eucalipto + pastagem; EPG: eucalipto + pastagem + gado; LVA: Latossolo Vermelho-Amarelo; LV: Latossolo Vermelho 
A avaliação final do IQS foi limitada às classes utilizadas por Melo Filho et al. (2009): IQS < 0,50 (ruim); $\mathrm{IQS}_{2}$ entre 0,50 a 0,70 (média); e $\mathrm{IQS}_{2} \geq 0,71$ (ótima). Assim, observa-se que nenhum dos sistemas instalados sobre os Latossolos Vermelho-Amarelos (LVA e LVA $_{2}$ ) foram enquadrados como $\mathrm{IQS}_{2}$ ruim, sendo que o $\mathrm{LVA}_{1}$ apresentou maior dificuldade em manter a conservação do solo em comparação ao LVA.

Os sistemas de eucalipto convencional (EC2), cerrado nativo (CN2) e eucalipto em nível (EN1) instalados sobre o LVA 1 estão enquadrados na classe ótima de $\mathrm{IQS}_{2}$, apresentando valores de 0,74;0,73 e 0,72 , respectivamente. Estes sistemas tendem a apresentar uma equivalência entre as funções principais do solo e indicam que o cultivo de eucalipto em escala comercial, quando realizado de forma adequada, pode proporcionar altos índices de qualidade do solo. Porém, conforme a Figura 2B, apenas o EC1 diferiu estatisticamente dos sistemas instalados no LVA, pois este sistema obteve menor valor das funções principais do solo, o que refletiu em reduzido $\mathrm{IQS}_{2}$. O EC1, juntamente com o CN1 e ECq, foram enquadrados como IQS 2 média.

Os sistemas de manejo cerrado nativo, eucalipto + pastagem, eucalipto + pastagem + gado, eucalipto convencional (CN4, EP, EPG e EC3), instalados sobre Latossolos Vermelhos $\left(\mathrm{LV}_{1}\right.$ e $\left.\mathrm{LV}_{2}\right)$ foram enquadrados como qualidade ruim do solo, visto que obtiveram $\mathrm{IQS}_{2}$ de 0,$48 ; 0,47 ; 0,46$; e 0,44 , respectivamente. Os sistemas CN4 e EC3 apresentaram menor capacidade de promover o crescimento de raízes em profundidade e armazenar/ suprir nutrientes para as plantas (Tabela 6), devido à baixa fertilidade destes solos, porém estas áreas apresentaram uma alta capacidade de manter a conservação do solo devido à ausência de revolvimento constante e de tráfego de máquinas e animais (Tabela 1). Os sistemas EP e EPG apresentaram redução de duas funções principais do solo, que são a capacidade de receber, armazenar e suprir água; e manter a conservação do solo (Tabela 6), devido principalmente à alta resistência do solo à penetração, alcançando valores de 4,63 e 5,74 MPa, respectivamente, influenciados pela coleta da soja e pisoteio do gado. Os sistemas instalados sobre o $\mathrm{LV}_{2}$ apresentaram $\mathrm{IQS}_{2}$ enquadrados como ruins e separados no grupo de menor IQS (Figura 2B), juntamente com o EN2 e EC1.

Melo Filho et al. (2007), trabalhando com Latossolo Amarelo coeso (LAx) dos Tabuleiros Costeiros, sob floresta nativa, encontraram valor de $\mathrm{IQS}_{2}$ de 0,46 , sendo este valor próximo ao encontrado neste estudo para o cerrado nativo (CN4) instalado em um Latossolo Vermelho da região Noroeste de Minas Gerais. Conforme os mesmos autores, a produção agrícola nessa classe de solo coesa deve ser realizada necessariamente com a melhoria da capacidade de retenção e armazenamento de água, redução da acidez e aumento da oferta de nutrientes. Melo Filho et al. (2009) determinaram o índice de qualidade para Latossolo Amarelo coeso cultivado com citros no sistema tradicional de uso e manejo para a região do Recôncavo Baiano e encontraram valor de 0,54 , sendo este enquadrado como regular, com limitações advindas da elevada resistência do solo à penetração, baixa permeabilidade à água e baixo teor de matéria orgânica, restringindo o crescimento e aprofundamento do sistema radicular e consequentemente o fornecimento e a disponibilidade de água para as plantas cítricas.

Neste trabalho, o IQS I $_{1}$ ostrou-se eficiente em indicar as deteriorações causadas no solo quando se converte os sistemas nativos em florestas plantadas, apresentando menor subjetividade e maior simplicidade de aplicação; porém o IQS foi eficiente em identificar quais são as funções principais do solo que estão em melhores e piores condições na situação de uso e manejo atuais apontando para uma possível melhoria nas situações mais críticas. Independente da metodologia adotada, os índices de qualidade do solo refletem o desempenho integrado dos atributos físicos e químicos dos solos estudados, e por isto eles são ferramentas úteis que poderão ser utilizadas nas tomadas de decisões sobre o sistema de manejo e uso em questão.

\section{CONCLUSÕES}

1. As áreas de manejo florestal sofreram reduções do IQS 1 quando comparados aos sistemas nativos, sendo que apenas os sistemas agrossilvopastoris apresentaram valores superiores de qualidade quando comparados aos sistemas de referência;

2. Os Latossolos Vermelho-Amarelos (LVA e LVA ${ }_{1}$ ) apresentaram qualidade superior aos Latossolos Vermelhos $\left(\mathrm{LV}_{1}\right.$ e $\left.\mathrm{LV}_{2}\right)$ quando se utiliza o IQS $\mathrm{I}_{2}$, devido às menores limitações dos atributos físicos e químicos nas funções principais dos solos, influenciadas pelos sistemas de uso e manejo daqueles solos;

3. Os sistemas agrossilvopastoris que utilizam eucalipto com arroz, eucalipto com soja e eucalipto com pastagem proporcionaram melhoria da qualidade do $\mathrm{LV}$, indicada pelo IQS ;

4. Os índices de qualidade dos Latossolos gerados, tanto com base nos desvios dos atributos do solo em relação aos sistemas naturais, como a partir do estabelecimento das funções do solo e indicadores a elas associados, foram eficientes em refletir a variação da qualidade do solo nos diferentes sistemas de uso e manejo florestal. 


\section{REFERÊNCIAS}

ALVARENGA, R. C. et al. Estabilidade de agregados de um Latossolo Roxo sob diferentes métodos de preparo do solo e de manejo da palhada do milho. Revista Brasileira de Ciência do Solo, v. 10, n. 02, p. 273-277, 1986.

ARAÚJO, R. et al. Qualidade de um solo sob diferentes usos e sob cerrado nativo. Revista Brasileira de Ciência do Solo, v. 31, n. 05, p. 1099-1108, 2007.

CARDOSO, E. L. Qualidade do solo em sistemas de pastagens cultivada e nativa na sub-região da Nhecolândia, Pantanal Sul Mato-Grossense. 2008. 154 f. Tese (Doutorado em Ciência do Solo) - Universidade Federal de Lavras, Lavras, 2008.

CARDOSO, E. L. et al. Atributos biológicos indicadores da qualidade do solo em pastagens cultivada e nativa no Pantanal. Pesquisa Agropecuária Brasileira, v. 44, n. 06, p. 631-637, 2009.

CARDOSO, E. L. et al. Qualidade química e física do solo sob vegetação arbórea nativa e pastagens no Pantanal Sul-MatoGrossense. Revista Brasileira de Ciência do Solo, v. 35, n. 02, p. 613-622, 2011.

CARVALHO, R. et al. Atributos físicos da qualidade de um solo sob sistema agroflorestal. Pesquisa Agropecuária Brasileira, v. 39, n. 11, p. 1153-1155, 2004.

CHAER, G. M.; TÓTOLA, M. R. Impacto do manejo de resíduos orgânicos durante a reforma de plantios de eucalipto sobre indicadores de qualidade do solo. Revista Brasileira de Ciência do Solo, v. 31, n. 06, p. 1381-1396, 2007.

CONCEIÇÃO, P. C. et al. Qualidade do solo em sistemas de manejo avaliada pela dinâmica da matéria orgânica e atributos relacionados. Revista Brasileira de Ciência do Solo, v. 29, n. 05, p. 777-788, 2005.

DANIELSON, R. E.; SUTHERLAND, P. L. Porosity. In: Klute, A. (Ed.). Methods of soil analysis. 2. ed. Madison: American Society of Agronomy, 1986, p. 443-461. v. 01.

DORAN, J. W.; PARKIN, T. B. Defining and assessing soil quality. In: DORAN, J. W. et al. Defining soil quality for sustainable environment. Madison: Soil Science Society of America Proceedings, 1994. p. 03-21.

EMPRESABRASILEIRADEPESQUISAAGROPECUÁRIA. Serviço Nacional de Levantamento e Conservação do Solo. Manual de análises de solo. 2. ed. Rio de Janeiro, 1997. $212 \mathrm{p}$.

FERREIRA, E. A. B. et al. Dinâmica do carbono da biomassa microbiana em cinco épocas do ano em diferentes sistemas de manejo do solo no cerrado. Revista Brasileira de Ciência do Solo, v. 31, n. 06, p. 1625-1635, 2007.

FIALHO, J. S. et al. Indicadores da qualidade do solo, em sistema de rotação, na Chapada do Apodi, Ceará. Revista Ciência Agronômica, v. 39, n. 03, p. 353-361, 2008.
FREITAS, D. A. F. Qualidade do solo em sistemas de manejo em Latossolos sob cerrado. 2010. 104 f. Dissertação (Mestrado em Ciência do Solo) - Universidade Federal de Lavras, Lavras, 2010.

GLOVER, J. D. et al. Systematic method for rating soil quality of conventional, organic, and integrated apple orchards in Washington State. Agriculture, Ecossystems and Environment, v. 80, n. 01/02, p. 29-45, 2000.

GRANT, C. A.; LANFOND, G. O. The effects of tillage systems and crop sequences on soil bulk density and penetration resistance on a clay soil in Southern Saskatchewan. Canadian Journal of Soil Science, v. 73, n. 02, p. 223-232, 1993.

ISLAM, K. R.; WEIL, R. R. Soil quality indicators properties in MidAtlantic soils as influenced by conservation management. Journal of Soil and Water Conservation, v. 55, n. 01, p. 69-78, 2000.

KARLEN, D. L.; STOTT, D. E. A framework for evaluating physics and chemical indicators of soil quality. In: DORAN, J.W. (Ed.). Defining soil quality for a sustainable environment. Madison: American Society of Agronomy, 1994. p. 53-71.

LEPSCH, I. F. Manual de levantamento utilitário do meio físico e classificação de terras no sistema de capacidade de uso. Viçosa, MG: Sociedade Brasileira de Ciência do Solo, 1983. 175 p.

LIMA, J. M. et al. Dispersão do material de solo em água para avaliação indireta da erodibilidade de latossolos. Revista Brasileira de Ciência do Solo, v. 14, n. 01, p. 85-90, 1990.

MAECHLER, M. et al. Cluster Analysis Basics and Extensions. Cluster R package, Viena, 2005.

MELLONI, R. et al. Avaliação da qualidade de solos sob diferentes coberturas florestais e de pastagem no sul de Minas Gerais. Revista Brasileira de Ciência do Solo, v. 32, n. 06, p. 2461-2470, 2008.

MELO FILHO, J. F. de. et al. Determinação do índice de qualidade subsuperficial em um Latossolo Amarelo Coeso dos Tabuleiros Costeiros, sob floresta natural. Revista Brasileira de Ciência do Solo, v. 31, n. 06, p. 1599-1608, 2007.

MELO FILHO, J. F de. et al. Índice de qualidade em um latossolo amarelo coeso cultivado com citros. Revista Brasileira de Fruticultura, v. 31, n. 04, p. 1168-1177, 2009.

MOTA, L. H. da S. de O.; VALLADARES, G. S. Vulnerabilidade à degradação dos solos da Bacia do Acaraú, Ceará. Revista Ciência Agronômica, v. 42, n. 01, p. 39-50, 2011.

NEVES, C. M. N. et al. Atributos indicadores da qualidade do solo em sistema agrossilvopastoril no noroeste do estado de Minas Gerais. Scientia Forestalis, v. 74, n. 02, p. 45-53, 2007.

RHEINHEIMER, D. S. et al. Modificações nos atributos químicos de solo sob campo nativo submetido à queima. Revista Ciência Rural, v. 33, n. 01, p. 49-55, 2003.

SILVEIRA, R. B. et al., Atributos microbiológicos e bioquímicos como indicadores da recuperação de áreas 
degradadas, em Itajubá/MG. Cerne, v. 12, n. 01, p. 48-55, 2006.

TÓTOLA, M. R.; CHAER, G.M. Microrganismos e processos microbiológicos como indicadores da qualidade dos solos. In: ALVAREZ V, V. H. et al. (Ed). Tópicos em ciência do solo. Viçosa, MG: Sociedade Brasileira de Ciência do Solo. 2002. p. 196-276. v. 02.
VEZZANI, F. M.; MIELNICZUK, J. Uma visão sobre qualidade do solo. Revista Brasileira de Ciência do Solo, v. 33, n. 04, p. 743-755, 2009.

WYMORE, A. W. Model-based systems engineering: an introduction to the mathematical theory of discrete systems and to the tricotyledon theory of system design. Boca Raton: CRC, 1993. $710 \mathrm{p}$. 